\title{
Critical Periods of Perturbations of Reversible Rigidly Isochronous Centers
}

\author{
Jiamei Zhou, ${ }^{1} \mathrm{Na} \mathrm{Li,}{ }^{1,2}$ and Maoan Han ${ }^{1}$ \\ ${ }^{1}$ The Institute of Mathematics, Shanghai Normal University, Shanghai 200234, China \\ ${ }^{2}$ Shanghai University of Engineering Science, Shanghai 201620, China
}

Correspondence should be addressed to Maoan Han; mahan@shnu.edu.cn

Received 29 March 2013; Accepted 10 May 2013

Academic Editor: Valery G. Romanovski

Copyright (C) 2013 Jiamei Zhou et al. This is an open access article distributed under the Creative Commons Attribution License, which permits unrestricted use, distribution, and reproduction in any medium, provided the original work is properly cited.

We study the problem of bifurcation of critical periods of a time-reversible polynomial system of degree $n$. We first present a new method to find the number of zeros of the period function. Then applying our results, we study the number of critical periods for some polynomial systems and obtain new results.

\section{Introduction}

Consider a two-dimensional analytic real differential system of the form

$$
\dot{x}=F(x, y), \quad \dot{y}=G(x, y),
$$

where we suppose there is a nondegenerate center at the origin $O$. Let $L(h)$ denote the orbit passing through the point $(h, 0)$ with $h>0$ of $(1)$ and $T(h)$ denote its period. As we all know, the isolated zeros of derivative of $T(h)$ are named critical periods. An interesting problem is to investigate the number of the critical periods. This is an important problem in the research of period functions and a lot of results have been obtained for polynomial differential systems, for example, monotonicity [1-8], finiteness of critical periods $[9,10]$ and isochronicity [11-13], and local bifurcation of critical periods [14]. The number of critical periods was also discussed in [15-17] for perturbations of isochronous vector fields.

Recently, the authors in [18] studied the following system:

$$
\begin{aligned}
& \dot{x}=-y+F_{n}(x, y)+\varepsilon \sum_{i=2}^{m} \widetilde{P}_{i}(x, y), \\
& \dot{y}=x+K_{n}(x, y)+\varepsilon \sum_{i=2}^{m} \widetilde{Q}_{i}(x, y),
\end{aligned}
$$

where $n, m \geq 2,0<\varepsilon \ll 1$,

$$
\begin{gathered}
F_{n}(x, y)=\sum_{j=1}^{[n / 2]} a_{n+1-2 j, 2 j-1} x^{n+1-2 j} y^{2 j-1}, \\
\widetilde{P}_{i}(x, y)=\sum_{j=1}^{[(i+1) / 2]} b_{i+1-2 j, 2 j-1} x^{i+1-2 j} y^{2 j-1}, \\
K_{n}(x, y)=\sum_{j=1}^{[n / 2]} a_{n+1-2 j, 2 j-1} x^{n-2 j} y^{2 j}, \\
\widetilde{Q}_{i}(x, y)=\sum_{j=1}^{[(i+2) / 2]} c_{i+2-2 j, 2 j-2} x^{i+2-2 j} y^{2 j-2} .
\end{gathered}
$$

Under the conditions above, system (2) is time-reversible and hence has a center at the origin.

As shown in [15-20], for $0<\varepsilon \ll 1$, the period function $\widetilde{T}(h, \varepsilon)$ of system (2) can be written as

$$
\widetilde{T}(h, \varepsilon)=T_{0}+\sum_{i=1}^{+\infty} \widetilde{T}_{i}(h) \varepsilon^{i}
$$

where $T_{0}$ is a positive constant. The authors [18] gave expressions of $\widetilde{T}_{1}(h)$ and $\widetilde{T}_{2}(h)$. It is also proved in [18] that one critical period can appear for the case $n=m=2$ and two critical periods can be found for the case $n=m=3$. 
In this paper, we consider the following system:

$$
\begin{aligned}
\dot{x}= & -y+\left[F_{n}(x, y)+\lambda \bar{F}_{l}(x, y)\right] \\
& +\varepsilon\left[P_{m}(x, y)+\lambda \bar{P}_{k}(x, y)\right], \\
\dot{y}= & x+\left[K_{n}(x, y)+\lambda \bar{K}_{l}(x, y)\right] \\
& +\varepsilon\left[Q_{m}(x, y)+\lambda \bar{Q}_{k}(x, y)\right],
\end{aligned}
$$

where

$$
\begin{gathered}
F_{n}(x, y)=\sum_{j=1}^{[n / 2]} a_{n+1-2 j, 2 j-1} x^{n-2 j+1} y^{2 j-1}, \\
\bar{F}_{l}(x, y)=\sum_{j=1}^{[l / 2]} \bar{a}_{l+1-2 j, 2 j-1} x^{l-2 j+1} y^{2 j-1}, \\
P_{m}(x, y)=\sum_{i=2}^{m} \sum_{j=1}^{[(i+1) / 2]} b_{i+1-2 j, 2 j-1} x^{i+1-2 j} y^{2 j-1}, \\
\bar{P}_{k}(x, y)=\sum_{i=2}^{k} \sum_{j=1}^{[(i+1) / 2]} \bar{b}_{i+1-2 j, 2 j-1} x^{i+1-2 j} y^{2 j-1}, \\
K_{n}(x, y)=\sum_{j=1}^{[n / 2]} a_{n+1-2 j, 2 j-1} x^{n-2 j} y^{2 j}, \\
\bar{Q}_{k}(x, y)=\sum_{i=2} \sum_{j=1}^{[(i+2) / 2]} \bar{c}_{i+2-2 j, 2 j-2} x^{i+2-2 j} y^{2 j-2}, \\
\sum_{j=1}^{[l / 2]} \bar{a}_{l+1-2 j, 2 j-1} x^{l-2 j} y^{2 j}, \\
\sum_{i=2}^{m} \sum_{j=1}^{[(i+2) / 2]} c_{i+2-2 j, 2 j-2} x^{i+2-2 j} y^{2 j-2},
\end{gathered}
$$

$m, n, l, k \geq 2$, and both $\varepsilon$ and $\lambda$ are small parameters. From the above expressions, we know that the system (5) satisfies the following properties: (i) it is a time-reversible system, and the perturbations with $\varepsilon$ are of general form; (ii) for all $\varepsilon$, the origin is a center, and for $\varepsilon=0$, the origin is isochronous since there exist polynomials $\varphi_{n}(x, y)$ and $\varphi_{l}(x, y)$ such that $F_{n}(x, y)=x \varphi_{n}(x, y), K_{n}(x, y)=y \varphi_{n}(x, y), \bar{F}_{l}(x, y)=$ $x \varphi_{l}(x, y), \bar{K}_{l}(x, y)=y \varphi_{l}(x, y)$.

Let $T(h, \varepsilon, \lambda)$ denote the period of the periodic orbit of system (5) passing through the point $(h, 0)$. Then it has the following expansion:

$$
T(h, \varepsilon, \lambda)=T_{0}+\sum_{i=1}^{+\infty} T_{i}(h, \lambda) \varepsilon^{i},
$$

where

$$
T_{i}(h, \lambda)=T_{i 0}(h)+\sum_{j=1}^{+\infty} T_{i j}(h) \lambda^{j}
$$

and since (5) becomes (2) as $\lambda=0$, we have $T(h, \varepsilon, 0)=$ $\widetilde{T}(h, \varepsilon)$.

Next section, we give some preliminary lemmas.

\section{Preliminary Lemmas}

In this section, we cite some results obtained in [18].

Lemma 1 (see [18]). For system (5),

$$
\begin{aligned}
T_{1}(h, 0) & =T_{10}(h) \\
& =-\sum_{i=1}^{m-1} \int_{0}^{2 \pi} \frac{h^{i} S_{i}(\theta)}{\left(1+(1-n) h^{n-1} \int_{0}^{\theta} G_{0}(\alpha) d \alpha\right)^{i /(n-1)}} d \theta \\
T_{2}(h, 0)= & T_{20}(h) \\
= & \int_{0}^{2 \pi}\left[\left(\sum_{i=1}^{m-1} r_{0}^{i}(\theta, h) S_{i}(\theta)\right)^{2}\right. \\
& \left.-\sum_{i=1}^{m-1} i r_{0}^{i-1}(\theta, h) r_{1}(\theta, h) S_{i}(\theta)\right] d \theta
\end{aligned}
$$

where

$$
\begin{aligned}
& r_{0}(\theta, h)=\left[h^{1-n}+(1-n) \int_{0}^{\theta} G_{0}(\alpha) d \alpha\right]^{1 /(1-n)} \\
& G_{0}(\theta)=\sum_{j=1}^{[n / 2]} a_{n+1-2 j, 2 j-1} \cos ^{n-2 j}(\theta) \sin ^{2 j-1}(\theta),
\end{aligned}
$$

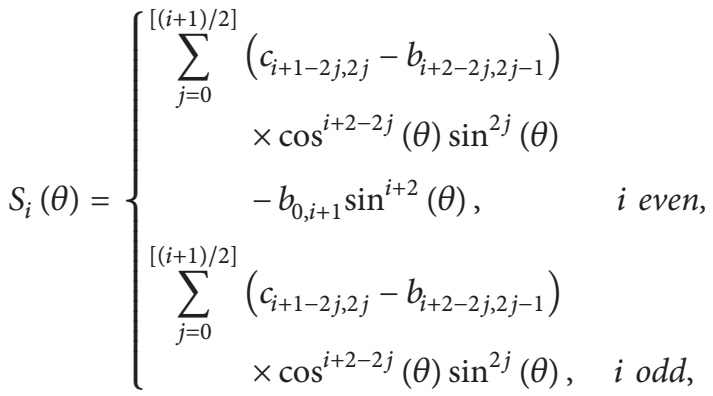

$$
\begin{aligned}
& r_{1}(\theta, h)=\int_{0}^{\theta}\left[\sum_{i=1}^{m-1} r_{0}^{i+1}(\alpha, h) G_{i}(\alpha)-G_{0}(\alpha)\right. \\
& \left.\times \sum_{i=1}^{m-1} r_{0}^{n+i}(\alpha, h) S_{i}(\alpha)\right] \\
& \cdot \exp \left(\int_{\alpha}^{\theta} n r_{0}^{n-1}(\beta, h) G_{0}(\beta) d \beta\right) d \alpha,
\end{aligned}
$$


moreover, if $n$ is odd, $S_{0}(\theta) \equiv 0$, and

$$
\begin{aligned}
& T_{1}(h, 0) \\
& =-\sum_{k=1}^{[(m-1) / 2]} \int_{0}^{2 \pi} \frac{h^{2 k} S_{2 k}(\theta)}{\left(1+(1-n) h^{n-1} \int_{0}^{\theta} G_{0}(\alpha) d \alpha\right)^{2 k /(n-1)}} d \theta .
\end{aligned}
$$

Lemma 2 (see [18]). Let $n=m=2$ and $\lambda=0$ in (5). Then

$$
\begin{aligned}
& T_{1}(h, 0) \\
& =\left(D_{02}-3 D_{20}\right) \pi \\
& \quad+\frac{2\left(D_{02}-D_{20}\right) \pi}{h^{2}}+\frac{4\left(D_{20}-D_{02}\right) \pi}{h} \\
& \quad+\frac{2(1-h)\left[D_{20} h^{2}+2\left(D_{02}-D_{20}\right) h+D_{20}-D_{02}\right] \pi}{h^{2} \sqrt{1-2 h}},
\end{aligned}
$$

where $h \in(0,1 / 2)$ and $D_{i j}$ are constants depending on the coefficients appearing in $F_{n}, K_{n}, P_{m}$, and $Q_{m}$. Furthermore, for sufficiently small $\varepsilon$,

(i) the center $O$ preserves the isochronicity when $D_{20}=$ $D_{02}=0$; period function $T(h, \varepsilon, 0)$ is increasing (resp., decreasing) for $h \in(0,1 / 2)$ when $D_{20}=0$ and $D_{02}>0$ (resp., <0);

(ii) there is at most one critical period in $(0,1 / 2)$ when $D_{20} \neq 0$. Moreover, there is exactly one critical period in $(0,1 / 2)$ if and only if

$$
r_{*}:=\frac{D_{02}}{D_{20}}<-3
$$

Lemma 3 (see [18]). Let $n=m=3$ and $\lambda=0$ in (5). Then

$$
\begin{aligned}
T_{1}(h, 0)= & \frac{1-\sqrt{1-h^{2}}}{\left(1+\sqrt{1-h^{2}}\right) \sqrt{1-h^{2}}} \pi \\
& \times\left[2 B_{03}-2 D_{30}+\left(B_{03}+B_{21}-D_{12}-D_{30}\right)\right. \\
& \left.\times \sqrt{1-h^{2}}+2 D_{30} h^{2}\right],
\end{aligned}
$$

where $h \in(0,1)$ and $B_{i j}, D_{i j}$ are constants depending on the coefficients appearing in $F_{n}, K_{n}, P_{m}$, and $Q_{m}$. Furthermore, for sufficiently small $\varepsilon$, one has the following results.

(i) The center $O$ preserves the isochronicity when

$$
\begin{gathered}
D_{20}=B_{11}-D_{02}=0, \\
D_{30}=B_{03}=B_{21}-D_{12}=0 .
\end{gathered}
$$

(ii) If (20) do not hold, there are at most two critical periods in $(0,1)$ and the maximum is achievable.

\section{Main Results}

As in [18], one can make the polar coordinates $x=r \cos (\theta)$, $y=r \sin (\theta)$, so that system (5) becomes

$$
\begin{aligned}
\dot{r}= & r^{n} G_{0}(\theta)+\lambda r^{l} \bar{G}_{0}(\theta) \\
& +\varepsilon\left[\sum_{i=1}^{m-1} r^{i+1} G_{i}(\theta)+\lambda \sum_{i=1}^{k-1} r^{i+1} \bar{G}_{i}(\theta)\right], \\
\dot{\theta} & =1+\varepsilon\left[\sum_{i=1}^{m-1} r^{i} S_{i}(\theta)+\lambda \sum_{i=1}^{k-1} r^{i} \bar{S}_{i}(\theta)\right],
\end{aligned}
$$

where $G_{0}(\theta)$ and $S_{i}(\theta)$ are given by (13) and (14),

$$
\bar{G}_{0}(\theta)=\sum_{j=1}^{[l / 2]} \bar{a}_{l+1-2 j, 2 j-1} \cos ^{l-2 j}(\theta) \sin ^{2 j-1}(\theta),
$$

$$
G_{i}(\theta)=\left\{\begin{array}{cl}
\sum_{j=1}^{[(i+2) / 2]}\left(b_{i+2-2 j, 2 j-1}+c_{i+3-2 j, 2 j-2}\right) & \\
& \times \cos ^{i+3-2 j}(\theta) \sin ^{2 j-1}(\theta), \quad i \text { even, } \\
\sum_{j=1}^{[(i+2) / 2]} & \left(b_{i+2-2 j, 2 j-1}+c_{i+3-2 j, 2 j-2}\right) \\
& \times \cos ^{i+3-2 j}(\theta) \sin ^{2 j-1}(\theta) \\
& +c_{0, i+1} \sin ^{i+2}(\theta), \quad i \text { odd }
\end{array}\right.
$$

$$
\bar{G}_{i}(\theta)=\left\{\begin{array}{cc}
\sum_{j=1}^{[(i+2) / 2]}\left(\bar{b}_{i+2-2 j, 2 j-1}+\bar{c}_{i+3-2 j, 2 j-2}\right) \\
\times \cos ^{i+3-2 j}(\theta) \sin ^{2 j-1}(\theta), \quad i \text { even, } \\
\sum_{j=1}^{[(i+2) / 2]}\left(\bar{b}_{i+2-2 j, 2 j-1}+\bar{c}_{i+3-2 j, 2 j-2}\right) \\
\times \cos ^{i+3-2 j}(\theta) \sin ^{2 j-1}(\theta) \\
+\bar{c}_{0, i+1} \sin ^{i+2}(\theta), \quad i \text { odd },
\end{array}\right.
$$

$$
\bar{S}_{i}(\theta)=\left\{\begin{array}{cl}
\sum_{j=0}^{[(i+1) / 2]} & \left(\bar{c}_{i+1-2 j, 2 j}-\bar{b}_{i+2-2 j, 2 j-1}\right) \\
& \times \cos ^{i+2-2 j}(\theta) \sin ^{2 j}(\theta) \\
& -\bar{b}_{0, i+1} \sin ^{i+2}(\theta), \quad i \text { even, } \\
\sum_{j=0}^{[(i+1) / 2]}\left(\bar{c}_{i+1-2 j, 2 j}-\bar{b}_{i+2-2 j, 2 j-1}\right) & \\
& \times \cos ^{i+2-2 j}(\theta) \sin ^{2 j}(\theta), \quad i \text { odd. }
\end{array}\right.
$$

Obviously, $\dot{\theta} \equiv 1$ when $\varepsilon=0$, which implies that the unperturbed system (5) $\left.\right|_{\varepsilon=0}$ has an isochronous center at $O$ which is called a rigidly or uniformly isochronous center. We have the following fundamental result. 
Theorem 4. Let (8) and (9) hold. Then, for system (5), one has

$$
\begin{aligned}
T_{11}(h)= & -\sum_{i=1}^{m-1} \int_{0}^{2 \pi} i r_{00}^{i-1}(\theta, h) r_{01}(\theta, h) S_{i}(\theta) d \theta \\
& -\sum_{i=1}^{k-1} \int_{0}^{2 \pi} r_{00}^{i}(\theta, h) \bar{S}_{i}(\theta) d \theta
\end{aligned}
$$

where

$$
\begin{aligned}
r_{00}(\theta, h)= & \frac{h}{\left[1+(1-n) h^{n-1} \int_{0}^{\theta} G_{0}(\alpha) d \alpha\right]^{1 /(n-1)}}, \\
r_{01}(\theta, h)= & \int_{0}^{\theta} r_{00}^{l}(\alpha, h) \bar{G}_{0}(\alpha) \\
& \cdot\left[\exp \int_{\alpha}^{\theta} n r_{00}^{n-1}(\beta, h) G_{0}(\beta) d \beta\right] d \alpha .
\end{aligned}
$$

Proof. We follow the idea of proving Lemma 1 which is Theorem 2.1 given in [18].

From (21), we have

$$
\begin{aligned}
\frac{d r}{d \theta}= & \left(r^{n} G_{0}(\theta)+\lambda r^{l} \bar{G}_{0}(\theta)\right. \\
& \left.+\varepsilon\left[\sum_{i=1}^{m-1} r^{i+1} G_{i}(\theta)+\lambda \sum_{i=1}^{k-1} r^{i+1} \bar{G}_{i}(\theta)\right]\right) \\
& \times\left(1+\varepsilon\left[\sum_{i=1}^{m-1} r^{i} S_{i}(\theta)+\lambda \sum_{i=1}^{k-1} r^{i} \bar{S}_{i}(\theta)\right]\right)^{-1} .
\end{aligned}
$$

Let $r(\theta, \varepsilon, \lambda, h)$ be the solution of the above equation satisfying the initial condition $r(0, \varepsilon, \lambda, h)=h$. It can be written as a series of $\varepsilon$

$$
r(\theta, \varepsilon, \lambda, h)=\sum_{i=0}^{+\infty} r_{i}(\theta, \lambda, h) \varepsilon^{i}
$$

where $r_{0}(0, \lambda, h)=h, r_{i}(0, \lambda, h)=0, i \geq 1$.

Substituting (30) into differential equation (29) and comparing the coefficients of $\varepsilon^{0}$, we have

$$
\frac{d}{d \theta} r_{0}(\theta, \lambda, h)=r_{0}^{n}(\theta, \lambda, h) G_{0}(\theta)+\lambda r_{0}^{l}(\theta, \lambda, h) \bar{G}_{0}(\theta) .
$$

We can write $r_{0}(\theta, \lambda, h)=r_{00}(\theta, h)+r_{01}(\theta, h) \lambda+\cdots$, where $r_{00}(0, h)=h, r_{0 i}(0, h)=0, i \geq 1$. Then, substituting $r_{0}(\theta, \lambda, h)$ into (31), then comparing the coefficients of $\lambda^{0}$ and $\lambda$, we can obtain

$$
\begin{gathered}
\frac{d r_{00}(\theta, h)}{d \theta}=r_{00}^{n}(\theta, h) G_{0}(\theta), \\
\frac{d}{d \theta} r_{01}(\theta, h)=n r_{00}^{n-1}(\theta, h) r_{01}(\theta, h) G_{0}(\theta)+r_{00}^{l}(\theta, h) \bar{G}_{0}(\theta) .
\end{gathered}
$$

Solving the above ODEs associated with the initial values $r_{00}(0, h)=h$ and $r_{01}(0, h)=0$, we can get (27) and (28). By (21) we have

$$
\begin{aligned}
T(h, \varepsilon, \lambda)= & \int_{0}^{2 \pi} \frac{1}{\dot{\theta}} d \theta \\
= & \int_{0}^{2 \pi} \frac{d \theta}{1+\varepsilon\left[\sum_{i=1}^{m-1} r^{i} S_{i}(\theta)+\lambda \sum_{i=1}^{k-1} r^{i} \bar{S}_{i}(\theta)\right]} \\
= & 2 \pi-\int_{0}^{2 \pi}\left[\sum_{i=1}^{m-1} r_{0}^{i}(\theta, \lambda, h) S_{i}(\theta)\right. \\
& +O\left(\varepsilon^{2}\right) .
\end{aligned}
$$

Therefore,

$$
\begin{aligned}
T_{1}(h, \lambda)= & -\int_{0}^{2 \pi}\left[\sum_{i=1}^{m-1} r_{0}^{i}(\theta, \lambda, h) S_{i}(\theta)\right. \\
& \left.+\lambda \sum_{i=1}^{k-1} r_{0}^{i}(\theta, \lambda, h) \bar{S}_{i}(\theta)\right] d \theta \\
= & -\sum_{i=1}^{m-1} \int_{0}^{2 \pi} r_{00}^{i}(\theta, h) S_{i}(\theta) d \theta \\
& -\left[\sum_{i=1}^{m-1} \int_{0}^{2 \pi} i r_{00}^{i-1}(\theta, h) r_{01}(\theta, h) S_{i}(\theta) d \theta\right. \\
& +O\left(\sum_{i=1}^{k-1} \int_{0}^{2 \pi} r_{00}^{i}(\theta, h) \bar{S}_{i}(\theta) d \theta\right] \lambda
\end{aligned}
$$

It implies that

$$
\begin{gathered}
T_{10}(h)=-\sum_{i=1}^{m-1} \int_{0}^{2 \pi} r_{00}^{i}(\theta, h) S_{i}(\theta) d \theta, \\
T_{11}(h)=-\left[\sum_{i=1}^{m-1} \int_{0}^{2 \pi} i r_{00}^{i-1}(\theta, h) r_{01}(\theta, h) S_{i}(\theta) d \theta\right. \\
\left.+\sum_{i=1}^{k-1} \int_{0}^{2 \pi} r_{00}^{i}(\theta, h) \bar{S}_{i}(\theta) d \theta\right] .
\end{gathered}
$$

Thus, (26) is proved. This ends the proof of Theorem 4.

With the same method as for $r_{01}(\theta, h)$, we can compute $r_{0 i}(\theta, h)$ for $i \geq 2$ and give an expression of $T_{1 i}(h)$ for $i \geq 2$ from the proof of Theorem 4 , which are omitted here.

Define

$$
M_{0}(h)=\frac{d T_{10}(h)}{d h}, \quad M_{1}(h)=\frac{d T_{11}(h)}{d h} .
$$

Another fundamental result is as follows. 
Theorem 5. Let $T(h, \varepsilon, \lambda)$ be defined as before with $h \in(0, K)$. Then for $0<|\varepsilon| \ll|\lambda| \ll 1$, one has the following.

(i) The period function $T(h, \varepsilon, \lambda)$ of system (5) is increasing (resp., decreasing) in $h \in(0, K)$ if $M_{0}(h)>0$ (resp., < 0) for all $h \in(0, K)$.

(ii) If $M_{0}(h)$ is not identically zero, the number of critical periods of $T(h, \varepsilon, \lambda)$ in $(0, K)$ is not more than the number of zeros (take the multiplicity into consideration) of $M_{0}(h)$ in $(0, K)$. And there are exactly $k$ critical periods if $M_{0}(h)$ has exactly $k$ simple positive zeros.

(iii) If $M_{0}(h) \equiv 0$, the number of critical periods of $T(h, \varepsilon$, $\lambda)$ in $(0, K)$ is not larger than the number of zeros of $M_{1}(h)$ in $(0, K)$. And $k$ critical periods can appear if $M_{1}(h)$ has $k$ simple zeros. Similarly, the period function $T(h, \varepsilon, \lambda)$ of (5) is increasing (resp., decreasing) in $h \in$ $(0, K)$ if $M_{1}(h)>0$ (resp., <0).

Proof. From (8) and (9)

$$
\begin{aligned}
\frac{\partial T(h, \varepsilon, \lambda)}{\partial h}=\sum_{i=1}^{+\infty} \frac{\partial T_{i}(h, \lambda)}{\partial h} \varepsilon^{i}=\varepsilon \Gamma(h, \varepsilon, \lambda), \\
\Gamma(h, \varepsilon, \lambda)=\frac{\partial T_{1}(h, \lambda)}{\partial h}+\sum_{i=1}^{+\infty} \frac{\partial T_{i+1}(h, \lambda)}{\partial h} \varepsilon^{i}, \\
\frac{\partial T_{1}(h, \lambda)}{\partial h}=\sum_{i=0}^{+\infty} \frac{d T_{1 i}(h)}{d h} \lambda^{i} \\
=M_{0}(h)+\sum_{i=1}^{+\infty} \frac{d T_{1 i}(h)}{d h} \lambda^{i} \\
=\Gamma_{1}(h, \lambda) .
\end{aligned}
$$

If $M_{0}(h)>0, h \in(0, K)$, then there exists a $\lambda_{0}$ such that for $0<|\lambda|<\lambda_{0}, \partial T_{1}(h, \lambda) / \partial h$ has the same sign with $d T_{10}(h) /$ $d h$. For $0<\varepsilon \ll \lambda$, the sign of the function $\partial T(h, \varepsilon, \lambda) / \partial h$ is the same as $\partial T_{1}(h, \lambda) / \partial h$. Then the conclusion (i) is proved.

Further, suppose that $h_{1}, h_{2}, \ldots, h_{k}$ are the $k$ zeros of $M_{0}(h)$ with the multiplicity $m_{1}, m_{2}, \ldots, m_{k}$, respectively. We only need to prove that the number of critical periods of $T(h, \varepsilon, \lambda)$ is less than or equal to $\sum_{j=1}^{k} m_{j}$. For the purpose, it suffices to prove that $\partial T(h, \varepsilon, \lambda) / \partial h$ has at most $\sum_{j=1}^{k} m_{j}$ roots. Thus, we only need to prove that $\partial T(h, \varepsilon, \lambda) / \partial h$ has at most $m_{j}$ zeros near $h_{j}, j=1,2, \ldots, k$. At first, we prove that $\partial T_{1}(h, \lambda) / \partial h$ has at most $m_{j}$ zeros near $h_{j}$ for $\lambda$ small. If it is not the case, then $\partial T_{1}(h, \lambda) / \partial h$ has at least $m_{j}+$ 1 zeros near $h_{j}$; that is, there exists $\lambda_{n} \rightarrow 0$ such that $\partial T_{1}\left(h, \lambda_{n}\right) / \partial h$ has at least $m_{j}+1$ zeros $h_{i, n}\left(i=1,2, \ldots, m_{j}+\right.$ 1) near $h_{j}$, where $h_{i, n} \rightarrow h_{j}(n \rightarrow \infty)$. By Rolls theorem, $\partial^{2} T_{1}\left(h, \lambda_{n}\right) / \partial h^{2}$ has at least $m_{j}$ zeros near $h_{j}, \ldots$, and $\partial^{m_{j}+1} T_{1}\left(h, \lambda_{n}\right) / \partial h^{m_{j}+1}$ has at least one zero $h_{i, n}^{*}$ near $h_{j}$. Thus, $\partial^{m_{j}+1} T_{1}\left(h_{i, n}^{*}, \lambda_{n}\right) / \partial h^{m_{j}+1}=0$. Letting $n \rightarrow \infty$, we have $\partial^{m_{j}+1} T_{1}\left(h_{j}, 0\right) / \partial h^{m_{j}+1}=\partial^{m_{j}} M_{0}\left(h_{j}\right) / \partial h^{m_{j}}=0$. This is a contradiction. Thus, $\partial T_{1}(h, \lambda) / \partial h$ has at most $m_{j}$ zeros near $h_{j}$. Using the same method, for sufficiently small $\varepsilon, \Gamma(h, \varepsilon, \lambda)$ or $\partial T / \partial h$ has at most $m_{j}$ zeros near $h_{j}$. Thus, $T(h, \varepsilon, \lambda)$ has at most $m_{j}$ critical periods for each $h_{j}$. Thus, we have proved the first part of (ii).

For the second part of (ii), assume that $h_{1}, h_{2}, \ldots, h_{k}$ are the $k$ simple zeros of $M_{0}(h)$; that is, $M_{0}\left(h_{j}\right)=0$ and $M_{0}^{\prime}\left(h_{j}\right) \neq 0, j=1,2, \ldots, k$. Then

$$
\begin{gathered}
\Gamma_{1}\left(h_{j}, 0\right)=\left.\frac{\partial T_{1}(h, \lambda)}{\partial h}\right|_{(h, \lambda)=\left(h_{j}, 0\right)}=0, \\
\left.\frac{\partial \Gamma_{1}(h, \lambda)}{\partial h}\right|_{(h, \lambda)=\left(h_{j}, 0\right)}=\left.\frac{\partial^{2} T_{1}(h, \lambda)}{\partial h^{2}}\right|_{(h, \lambda)=\left(h_{j}, 0\right)} \neq 0 .
\end{gathered}
$$

By the Implicit Function Theorem, there is a unique function $h_{j}(\lambda)$ such that $\Gamma_{1}\left(h_{j}(\lambda), \lambda\right)=0$ and $h_{j}(0)=h_{j}, j=$ $1,2, \ldots, k$. Therefore $h_{1}(\lambda), h_{2}(\lambda), \ldots, h_{k}(\lambda)$ are $k$ simple zeros of $\partial T_{1}(h, \lambda) / \partial h$. By the same method, there is a unique function $h_{j}^{*}(\varepsilon, \lambda)$ such that $\Gamma\left(h_{j}^{*}(\varepsilon, \lambda), \varepsilon\right)=0$, and $h_{j}^{*}(0, \lambda)=$ $h_{j}(\lambda)$. The second part of (ii) is proved.

When $M_{0} \equiv 0$, then

$$
\begin{aligned}
\frac{\partial T_{1}(h, \lambda)}{\partial h} & =\Gamma_{1}(h, \lambda)=\sum_{i=1}^{+\infty} \frac{d T_{1 i}(h)}{d h} \lambda^{i} \\
& =\frac{d T_{11}(h)}{d h} \lambda+\sum_{i=2}^{+\infty} \frac{d T_{1 i}(h)}{d h} \lambda^{i} \\
& =\lambda \widetilde{\Gamma}_{1}(h, \lambda), \\
\widetilde{\Gamma}_{1}(h, \lambda) & =M_{1}(h)+\sum_{i=1}^{+\infty} \frac{d T_{1, i+1}(h)}{d h} \lambda^{i} .
\end{aligned}
$$

Then, we can prove conclusion (iii) in the same way as proving conclusion (ii). The proof is completed.

\section{Application}

In this section, we apply Theorems 4 and 5 to the cases $n=$ $m=2, l=k=3 ; n=m=3, l=k=4$, and $n=l=2$, $m=k=3$, respectively.

Case 1 ( $n=m=2, l=k=3$ ). In this case, system (5) becomes

$$
\begin{aligned}
\dot{x}= & -y+a_{11} x y+\lambda \bar{a}_{21} x^{2} y \\
& +\varepsilon\left[b_{11} x y+\lambda\left(\bar{b}_{11} x y+\bar{b}_{21} x^{2} y+\bar{b}_{03} y^{3}\right)\right], \\
\dot{y}= & x+a_{11} y^{2}+\lambda \bar{a}_{21} x y^{2} \\
& +\varepsilon\left[c_{20} x^{2}+c_{02} y^{2}\right. \\
& \left.+\lambda\left(\bar{c}_{20} x^{2}+\bar{c}_{02} y^{2}+\bar{c}_{30} x^{3}+\bar{c}_{12} x y^{2}\right)\right],
\end{aligned}
$$

where $a_{11} \neq 0$. For system (40), $O$ is a center because of the symmetry.

We only need to consider the case of $a_{11}>0$ in (40); otherwise, we can use the transformation $x \rightarrow-x, y \rightarrow-y$ to change (40) into the same form with opposite signs to 
the coefficients. Further, suppose $a_{11}=1$, otherwise, system (40) can be simplified as the form of system (41) by the transformation $u=a_{11} x, v=a_{11} y$. In this case, system (40) becomes

$$
\begin{aligned}
\dot{x}= & -y+x y+\lambda \bar{a}_{21} x^{2} y \\
& +\varepsilon\left[b_{11} x y+\lambda\left(\bar{b}_{11} x y+\bar{b}_{21} x^{2} y+\bar{b}_{03} y^{3}\right)\right], \\
\dot{y}= & x+y^{2}+\lambda \bar{a}_{21} x y^{2} \\
& +\varepsilon\left[c_{20} x^{2}+c_{02} y^{2}\right. \\
& \left.+\lambda\left(\bar{c}_{20} x^{2}+\bar{c}_{02} y^{2}+\bar{c}_{30} x^{3}+\bar{c}_{12} x y^{2}\right)\right] .
\end{aligned}
$$

It is easy to conclude that system (41) $\left.\right|_{\varepsilon=0}$ has a first integral of the form

$$
H(x, y)=\frac{2\left(1+\lambda \bar{a}_{21}\right) x+\left(1+\lambda \bar{a}_{21}\right) y^{2}-1}{\left(1+\lambda \bar{a}_{21}\right)^{2}\left[1-\left(1+\lambda \bar{a}_{21}\right) x\right]^{2}}
$$

Theorem 6. For system (41), one has

$$
\begin{aligned}
T_{10}(h)= & \left(c_{02}-b_{11}-3 c_{20}\right) \pi \\
& +\frac{2\left(c_{02}-b_{11}-c_{20}\right) \pi}{h^{2}}+\frac{4\left(c_{20}-c_{02}+b_{11}\right) \pi}{h} \\
& +\left(2 ( 1 - h ) \left[c_{20} h^{2}+2\left(c_{02}-b_{11}-c_{20}\right) h\right.\right. \\
& \left.\left.+c_{20}-c_{02}+b_{11}\right] \pi\right) \\
& \times\left(h^{2} \sqrt{1-2 h}\right)^{-1},
\end{aligned}
$$

where $h \in(0,1 / 2)$. For sufficiently small $\varepsilon$ and $\lambda$, there is at most one critical period if $M_{0}(h)$ is not identically zero and $c_{20} \neq 0$; there is exactly one critical period if and only if $\rho:=\left(c_{02}-b_{11}\right) / c_{20}<-3$. Otherwise, the period function $T(h, \varepsilon, \lambda)$ is increasing for $h \in(0,1 / 2)$ (resp., decreasing) if $c_{20}>0$ (resp., $\left.<0\right)$;

(ii) if $M_{0}(h)=T_{10}^{\prime}(h) \equiv 0$, then for $0<h \ll 1$,

$$
\begin{aligned}
T_{11}(h)=-[ & A_{2} h^{2}+A_{3} h^{3}+A_{4} h^{4} \\
& \left.+A_{5} h^{5}+A_{6} h^{6}+O\left(h^{6}\right)\right] \pi,
\end{aligned}
$$

where

$$
\begin{gathered}
A_{2}=-\frac{1}{4}\left(\bar{c}_{02}-\bar{b}_{11}\right)-\frac{3}{4} \bar{c}_{20}+\frac{3}{4} \bar{c}_{30} \\
+\frac{1}{4}\left(\bar{c}_{12}-\bar{b}_{11}\right)-\frac{3}{4} \bar{b}_{03}, \\
A_{3}=2 A_{2},
\end{gathered}
$$

$$
\begin{aligned}
A_{4}= & -\frac{23}{8} \bar{c}_{20}+\frac{33}{8} \bar{c}_{30}-\frac{7}{8}\left(\bar{c}_{02}-\bar{b}_{11}\right) \\
& -\frac{21}{8} \bar{b}_{03}+\frac{9}{8}\left(\bar{c}_{12}-\bar{b}_{21}\right), \\
A_{5}= & -\frac{9}{2} \bar{b}_{03}-\frac{11}{2} \bar{c}_{20}+\frac{5}{2}\left(\bar{c}_{12}-\bar{b}_{21}\right) \\
& +\frac{21}{2} \bar{c}_{30}-\frac{3}{2}\left(\bar{c}_{02}-\bar{b}_{11}\right), \\
A_{6}=- & \frac{675}{64} \bar{c}_{20}-\frac{99}{16} \bar{b}_{03}-\frac{165}{64}\left(\bar{c}_{02}-\bar{b}_{11}\right) \\
+ & \frac{323}{16} \bar{c}_{30}+\frac{69}{16}\left(\bar{c}_{12}-\bar{b}_{21}\right) .
\end{aligned}
$$

There can appear three critical periods in $h \in(0,1 / 2)$ if $T_{11}(h)$ is not identically zero.

Proof. For system (41), from (13), (14), (22)-(25), we have

$$
\begin{gathered}
G_{0}(\theta)=\sin (\theta), \quad \bar{G}_{0}(\theta)=\bar{a}_{21} \cos (\theta) \sin (\theta), \\
G_{1}(\theta)=\left(c_{20}+b_{11}\right) \cos ^{2}(\theta) \sin (\theta)+c_{02} \sin ^{3}(\theta), \\
\bar{G}_{1}(\theta)=\left(\bar{b}_{11}+\bar{c}_{20}\right) \cos ^{2}(\theta) \sin (\theta)+\bar{c}_{02} \sin ^{3}(\theta), \\
\bar{G}_{2}(\theta)=\left(\bar{b}_{21}+\bar{c}_{30}\right) \cos ^{3}(\theta) \sin (\theta) \\
+\left(\bar{b}_{03}+\bar{c}_{12}\right) \cos (\theta) \sin ^{3}(\theta), \\
S_{1}(\theta)=c_{20} \cos ^{3}(\theta)+\left(c_{02}-b_{11}\right) \cos (\theta) \sin ^{2}(\theta), \\
\bar{S}_{1}(\theta)=\bar{c}_{20} \cos ^{3}(\theta)+\left(\bar{c}_{02}-\bar{b}_{11}\right) \cos (\theta) \sin ^{2}(\theta), \\
\bar{S}_{2}(\theta)=\bar{c}_{30} \cos ^{4}(\theta)+\left(\bar{c}_{12}-\bar{b}_{21}\right) \cos { }^{2}(\theta) \sin ^{2}(\theta) \\
-\bar{b}_{03} \sin ^{4}(\theta) .
\end{gathered}
$$

From (27), we have

$$
r_{00}(\theta, h)=\frac{h}{1-h+h \cos (\theta)} .
$$

From (10), we have

$T_{10}(h)$

$$
\begin{aligned}
& =-\sum_{i=1}^{m-1} \int_{0}^{2 \pi} \frac{h^{i} S_{i}(\theta)}{\left[1+(1-n) h^{n-1} \int_{0}^{\theta} G_{0}(\alpha) d \alpha\right]^{i /(n-1)}} d \theta \\
& =-\int_{0}^{2 \pi} \frac{h S_{1}(\theta)}{1-h \int_{0}^{\theta} \sin (\alpha) d \alpha} d \theta \\
& =-\int_{0}^{2 \pi} \frac{h\left[c_{20} \cos ^{3}(\theta)+\left(c_{02}-b_{11}\right) \cos (\theta) \sin ^{2}(\theta)\right]}{1-h+h \cos (\theta)} d \theta
\end{aligned}
$$




$$
\begin{aligned}
= & -\int_{0}^{2 \pi} \frac{h \cos (\theta)}{1-h+h \cos (\theta)} \\
& \quad \times\left[c_{20} \cos ^{2}(\theta)+\left(c_{02}-b_{11}\right) \sin ^{2}(\theta)\right] d \theta \\
= & -\int_{0}^{2 \pi}\left(1-\frac{1-h}{1-h+h \cos (\theta)}\right) \\
& \quad \times\left[c_{20} \cos ^{2}(\theta)+\left(c_{02}-b_{11}\right) \sin ^{2}(\theta)\right] d \theta \\
= & \left(c_{02}-b_{11}-3 c_{20}\right) \pi+\frac{2\left(c_{02}-b_{11}-c_{20}\right) \pi}{h^{2}} \\
& +\frac{4\left(c_{20}-c_{02}+b_{11}\right) \pi}{h} \\
& +\frac{2(1-h)\left[c_{20} h^{2}+2\left(c_{02}-b_{11}-c_{20}\right) h+c_{20}+b_{11}-c_{02}\right] \pi}{h^{2} \sqrt{1-2 h}} .
\end{aligned}
$$

Then, from [18], we have

$$
\frac{d T_{10}(h)}{d h}=\frac{(1-\delta) F(\delta) \pi}{\delta^{3}(1-\delta)^{3}},
$$

where $\delta=\sqrt{1-2 h}$ and

$$
\begin{aligned}
F(\delta)= & c_{20} \delta^{4}+4 c_{20} \delta^{3}+\left(2 c_{20}+4 c_{02}-4 b_{11}\right) \delta^{2} \\
& +4 c_{20} \delta+c_{20} .
\end{aligned}
$$

Support that $c_{20} \neq 0$. By (50) we have four zeros of $F(\delta)$ as follows:

$$
\begin{aligned}
& \delta_{ \pm}=-1-\sqrt{1-\rho} \pm \sqrt{1-\rho-2 \sqrt{1-\rho}}, \\
& \bar{\delta}_{ \pm}=-1+\sqrt{1-\rho} \pm \sqrt{1-\rho-2 \sqrt{1-\rho}},
\end{aligned}
$$

where $\rho$ is given by $\rho=\left(c_{02}-b_{11}\right) / c_{20}$. It is not difficult to examine that $\delta_{ \pm}$is not in $(0,1)$ and $\bar{\delta}_{+}$is not in $(0,1)$. In addition, $\bar{\delta}_{-} \in(0,1)$ if and only if $\rho<-3$. Thus, there is at most one critical period in $(0,1 / 2)$. If $\rho \geq-3, F(\delta)$ has no zero in $h \in(0,1 / 2)$. From the expression of $d T_{10}(h) / d h$, we can easily check that $d T_{10}(h) / d h>0$ (resp., $<0$ ) when $c_{20}>0$ (resp., $<0$ ). Therefore, for sufficiently small $\varepsilon$ and $\lambda$, $\partial T(h, \varepsilon, \lambda) / \partial h>0$ (resp., $<0)$ if $c_{20}>0$ (resp., $\left.<0\right)$. The proof of conclusion (i) is completed.

For conclusion (ii), let $M_{0}(h) \equiv 0$, which gives $c_{20}=c_{02}-$ $b_{11}=0$ or $S_{1}(\theta)=0$. From (26), we have that

$$
\begin{aligned}
& T_{11}(h) \\
& =-\int_{0}^{2 \pi} r_{00}(\theta, h) \bar{S}_{1}(\theta) d \theta-\int_{0}^{2 \pi} r_{00}^{2}(\theta, h) \bar{S}_{2}(\theta) d \theta \\
& =-\int_{0}^{2 \pi} \frac{h\left[\bar{c}_{20} \cos ^{3}(\theta)+\left(\bar{c}_{02}-\bar{b}_{11}\right) \cos (\theta) \sin ^{2}(\theta)\right]}{1-h+h \cos (\theta)} d \theta \\
& -\int_{0}^{2 \pi}\left(\left(h ^ { 2 } \left[\bar{c}_{30} \cos ^{4}(\theta)+\left(\bar{c}_{12}-\bar{b}_{21}\right) \cos ^{2}(\theta) \sin ^{2}(\theta)\right.\right.\right. \\
& \left.\left.\left.-\bar{b}_{03} \sin ^{4}(\theta)\right]\right)(1-h+h \cos (\theta))^{-2}\right) d \theta .
\end{aligned}
$$

Then we expand $T_{11}(h)$ at $h=0$ by letting

$$
\begin{aligned}
T_{11}(h)=- & {\left[A_{2} h^{2}+A_{3} h^{3}+A_{4} h^{4}\right.} \\
& \left.+A_{5} h^{5}+A_{6} h^{6}+O\left(h^{6}\right)\right] \pi .
\end{aligned}
$$

By (52), we have

$$
\begin{aligned}
A_{2}= & -\frac{1}{4}\left(\bar{c}_{02}-\bar{b}_{11}\right)-\frac{3}{4} \bar{c}_{20}+\frac{3}{4} \bar{c}_{30} \\
& +\frac{1}{4}\left(\bar{c}_{12}-\bar{b}_{21}\right)-\frac{3}{4} \bar{b}_{03}, \\
A_{3}=2 A_{2}, & \\
A_{4}= & -\frac{23}{8} \bar{c}_{20}+\frac{33}{8} \bar{c}_{30}-\frac{7}{8}\left(\bar{c}_{02}-\bar{b}_{11}\right)-\frac{21}{8} \bar{b}_{03} \\
& +\frac{9}{8}\left(\bar{c}_{12}-\bar{b}_{21}\right), \\
A_{5}= & -\frac{9}{2} \bar{b}_{03}-\frac{11}{2} \bar{c}_{20}+\frac{5}{2}\left(\bar{c}_{12}-\bar{b}_{21}\right)+\frac{21}{2} \bar{c}_{30} \\
& -\frac{3}{2}\left(\bar{c}_{02}-\bar{b}_{11}\right), \\
A_{6}= & -\frac{675}{64} \bar{c}_{20}-\frac{99}{16} \bar{b}_{03}-\frac{165}{64}\left(\bar{c}_{02}-\bar{b}_{11}\right)+\frac{323}{16} \bar{c}_{30} \\
+ & \frac{69}{16}\left(\bar{c}_{12}-\bar{b}_{21}\right) .
\end{aligned}
$$

Note that

$$
\begin{aligned}
T_{11}^{\prime}(h)=-[ & 2 A_{2} h+3 A_{3} h^{2}+4 A_{4} h^{3}+5 A_{5} h^{4} \\
& \left.+6 A_{6} h^{5}+O\left(h^{5}\right)\right] \pi
\end{aligned}
$$

and that

$$
\begin{aligned}
& \operatorname{det} \frac{\partial\left(A_{2}, A_{4}, A_{5}, A_{6}\right)}{\partial\left(\bar{c}_{20}, \bar{c}_{02}-\bar{b}_{11}, \bar{b}_{03}, \bar{c}_{12}-\bar{b}_{21}\right)} \\
& =\left|\begin{array}{rrrr}
-\frac{3}{4} & -\frac{23}{8} & -\frac{3}{4} & -\frac{675}{64} \\
-\frac{1}{4} & -\frac{7}{8} & -\frac{21}{8} & -\frac{165}{64} \\
\frac{3}{4} & \frac{33}{8} & -\frac{9}{2} & \frac{323}{16} \\
\frac{1}{4} & \frac{9}{8} & -\frac{99}{16} & \frac{69}{16}
\end{array}\right|=\frac{41175}{16384} \neq 0 .
\end{aligned}
$$

Therefore, $A_{2}, A_{4}, A_{5}, A_{6}$ can be taken as free parameters. Hence, we can change the sign of $A_{2}, A_{4}, A_{5}, A_{6}$, satisfying

$$
0<-A_{2} \ll A_{4} \ll-A_{5} \ll A_{6},
$$

which ensures that $T_{11}^{\prime}(h)$ has three positive zeros in $h$ near $h=0$. Thus, conclusion (ii) is proved.

We remark that for cubic system (40), we obtain three critical periods using $T_{11}(h)$, which is one more than the results in Lemma 2.3 obtained in [18]. 
Case $2(n=m=3, l=k=4)$. In this case, system (40) becomes

$$
\begin{aligned}
& \dot{x}=-+a_{21} x^{2} y+\lambda \bar{a}_{31} x^{3} y+\lambda \bar{a}_{13} x y^{3} \\
&+ \varepsilon\left[b_{11} x y+b_{21} x^{2} y+b_{03} y^{3}\right. \\
&+\lambda\left(\bar{b}_{11} x y+\bar{b}_{21} x^{2} y+\bar{b}_{03} y^{3}\right. \\
&\left.\left.+\bar{b}_{31} x^{3} y+\bar{b}_{13} x y^{3}\right)\right], \\
& \dot{y}=x+ a_{21} x y^{2}+\lambda \bar{a}_{31} x^{2} y^{2}+\lambda \bar{a}_{13} y^{4} \\
&+\varepsilon\left[c_{20} x^{2}+c_{02} y^{2}+c_{30} x^{3}+c_{12} x y^{2}\right. \\
&+\lambda\left(\bar{c}_{20} x^{2}+\bar{c}_{02} y^{2}+\bar{c}_{30} x^{3}\right. \\
&\left.\left.+\bar{c}_{12} x y^{2}+\bar{c}_{40} x^{4}+\bar{c}_{22} x^{2} y^{2}+\bar{c}_{04} y^{4}\right)\right],
\end{aligned}
$$

where we suppose $a_{21} \neq 0$. This is a new system which is not studied in [18]. For the case $a_{21}>0$, by the rescaling

$$
u=\sqrt{a_{21}} x, \quad v=\sqrt{a_{21}} y,
$$

system (58) can be written as

$$
\begin{aligned}
& \dot{u}=-v+ u^{2} v+\lambda \bar{A}_{31} u^{3} v+\lambda \bar{A}_{13} u v^{3} \\
&+\varepsilon\left[B_{11} u v+B_{21} u^{2} v+B_{03} v^{3}\right. \\
&+\lambda\left(\bar{B}_{11} u v+\bar{B}_{21} u^{2} v+\bar{B}_{03} v^{3}\right. \\
&\left.\left.+\bar{B}_{31} u^{3} v+\bar{B}_{13} u v^{3}\right)\right] \\
& \dot{v}=u+u v^{2}+\lambda \bar{A}_{31} u^{2} v^{2}+\lambda \bar{A}_{13} v^{4} \\
&+\varepsilon\left[D_{20} u^{2}+D_{02} v^{2}+D_{30} u^{3}+D_{12} u v^{2}\right. \\
&+\lambda\left(\bar{D}_{20} u^{2}+\bar{D}_{02} v^{2}+\bar{D}_{30} u^{3}+\bar{D}_{12} u v^{2}\right. \\
&\left.\left.+\bar{D}_{40} u^{4}+\bar{D}_{22} u^{2} v^{2}+\bar{D}_{04} v^{4}\right)\right],
\end{aligned}
$$

where

$$
\begin{gathered}
\bar{A}_{31}=\frac{\bar{a}_{31}}{a_{21} \sqrt{a_{21}}}, \quad \bar{A}_{13}=\frac{\bar{a}_{13}}{a_{21} \sqrt{a_{21}}}, \quad B_{11}=\frac{b_{11}}{\sqrt{a_{21}}}, \\
B_{21}=\frac{b_{21}}{a_{21}}, \quad B_{03}=\frac{b_{03}}{a_{21}}, \quad \bar{B}_{11}=\frac{\bar{b}_{11}}{\sqrt{a_{21}}}, \\
\bar{B}_{21}=\frac{\bar{b}_{21}}{a_{21}}, \quad \bar{B}_{03}=\frac{\bar{b}_{03}}{a_{21}}, \quad \bar{B}_{31}=\frac{\bar{b}_{31}}{a_{21} \sqrt{a_{21}}}, \\
\bar{B}_{13}=\frac{\bar{b}_{13}}{a_{21} \sqrt{a_{21}}}, \quad D_{20}=\frac{c_{20}}{\sqrt{a_{21}}}, \quad D_{02}=\frac{c_{02}}{\sqrt{a_{21}}}, \\
D_{30}=\frac{c_{30}}{a_{21}}, \quad D_{12}=\frac{c_{12}}{a_{21}}, \quad \bar{D}_{20}=\frac{\bar{c}_{20}}{\sqrt{a_{21}}},
\end{gathered}
$$

$$
\begin{array}{cc}
\bar{D}_{02}=\frac{\bar{c}_{02}}{\sqrt{a_{21}}}, \quad \bar{D}_{30}=\frac{\bar{c}_{30}}{a_{21}}, \quad \bar{D}_{12}=\frac{\bar{c}_{12}}{a_{21}}, \\
\bar{D}_{40}=\frac{\bar{c}_{40}}{a_{21} \sqrt{a_{21}}}, \quad \bar{D}_{22}=\frac{\bar{c}_{22}}{a_{21} \sqrt{a_{21}}}, \quad \bar{D}_{04}=\frac{\bar{c}_{04}}{a_{21} \sqrt{a_{21}}} .
\end{array}
$$

If $a_{21}<0$, system (58) can be simplified as (60) similarly by the change $u=\sqrt{-a_{21}} x, v=\sqrt{-a_{21}} y$.

Theorem 7. For system (60), one has

$$
\begin{aligned}
T_{10}(h)= & \frac{1-\sqrt{1-h^{2}}}{\left(1+\sqrt{1-h^{2}}\right) \sqrt{1-h^{2}}} \pi \\
& \times\left[2 B_{03}-2 D_{30}+\left(B_{03}+B_{21}-D_{12}-D_{30}\right)\right. \\
& \left.\quad \times \sqrt{1-h^{2}}+2 D_{30} h^{2}\right]
\end{aligned}
$$

where $h \in(0,1)$. If $M_{0}(h)$ is not identically zero, there are at most two critical periods in $h \in(0,1)$ for sufficiently small $\varepsilon$ and $\lambda$, and the maximum can be achievable;

(ii) if $M_{0}(h) \equiv 0$, one has

$$
\begin{aligned}
T_{11}(h)= & \frac{1-\sqrt{1-h^{2}}}{\left(1+\sqrt{1-h^{2}}\right) \sqrt{1-h^{2}}} \pi \\
& \times\left[2 \bar{B}_{03}-2 \bar{D}_{30}+\left(\bar{B}_{03}+\bar{B}_{21}-\bar{D}_{12}-\bar{D}_{30}\right)\right. \\
& \left.\quad \times \sqrt{1-h^{2}}+2 \bar{D}_{30} h^{2}\right]
\end{aligned}
$$

and there are at most two critical periods in $h \in(0,1)$ if $T_{11}(h)$ is not identically zero.

Proof. From (13), (14), (22)-(25), we have that for system (60)

$$
\begin{gathered}
G_{0}(\theta)=\cos (\theta) \sin (\theta), \\
\bar{G}_{0}(\theta)=\bar{A}_{31} \cos ^{2}(\theta) \sin (\theta)+\bar{A}_{13} \sin ^{3}(\theta), \\
G_{1}(\theta)=\left(B_{11}+D_{20}\right) \cos ^{2}(\theta) \sin (\theta)+D_{02} \sin ^{3}(\theta), \\
G_{2}(\theta)=\left(B_{21}+D_{30}\right) \cos ^{3}(\theta) \sin (\theta) \\
+\left(B_{03}+D_{12}\right) \cos (\theta) \sin ^{3}(\theta), \\
\bar{G}_{1}(\theta)=\left(\bar{B}_{11}+\bar{D}_{20}\right) \cos ^{2}(\theta) \sin (\theta)+\bar{D}_{02} \sin ^{3}(\theta), \\
\bar{G}_{2}(\theta)=\left(\bar{B}_{21}+\bar{D}_{30}\right) \cos ^{3}(\theta) \sin (\theta) \\
+\left(\bar{B}_{03}+\bar{D}_{12}\right) \cos (\theta) \sin ^{3}(\theta),
\end{gathered}
$$




$$
\begin{aligned}
S_{1}(\theta)= & D_{20} \cos ^{3}(\theta)+\left(D_{02}-B_{11}\right) \cos (\theta) \sin ^{2}(\theta), \\
S_{2}(\theta)= & D_{30} \cos ^{4}(\theta)+\left(D_{12}-B_{21}\right) \cos ^{2}(\theta) \sin ^{2}(\theta) \\
& -B_{03} \sin ^{4}(\theta) \\
\bar{S}_{1}(\theta)= & \bar{D}_{20} \cos ^{3}(\theta)+\left(\bar{D}_{02}-\bar{B}_{11}\right) \cos (\theta) \sin ^{2}(\theta), \\
\bar{S}_{2}(\theta)= & \bar{D}_{30} \cos ^{4}(\theta)+\left(\bar{D}_{12}-\bar{B}_{21}\right) \cos ^{2}(\theta) \sin ^{2}(\theta) \\
& -\bar{B}_{03} \sin ^{4}(\theta), \\
\bar{S}_{3}(\theta)= & \bar{D}_{40} \cos ^{5}(\theta)+\left(\bar{D}_{22}-\bar{B}_{31}\right) \cos ^{3}(\theta) \sin ^{2}(\theta) \\
& +\left(\bar{D}_{04}-\bar{B}_{13}\right) \sin ^{4}(\theta) \cos (\theta) .
\end{aligned}
$$

From (27) and (28), we have

$$
\begin{gathered}
r_{00}(\theta, h)=\frac{h}{\sqrt{1-h^{2} \sin ^{2}(\theta)}}, \\
r_{01}(\theta, h)=r_{00}^{3}(\theta, h) \frac{1-\sqrt{1-h^{2} \sin ^{2}(\theta)}}{h} .
\end{gathered}
$$

Hence, from (10),

$$
\begin{aligned}
& T_{10}(h) \\
& =-\sum_{i=1}^{m-1} \int_{0}^{2 \pi} \frac{h^{i} S_{i}(\theta)}{\left[1+(1-n) h^{n-1} \int_{0}^{\theta} G_{0}(\alpha) d \alpha\right]^{i /(n-1)}} d \theta \\
& =-\int_{0}^{2 \pi} \frac{h S_{1}(\theta)}{\sqrt{1-h^{2} \sin ^{2}(\theta)}} d \theta-\int_{0}^{2 \pi} \frac{h^{2} S_{2}(\theta)}{1-h^{2} \sin ^{2}(\theta)} d \theta \\
& =\frac{1-\sqrt{1-h^{2}}}{\left(1+\sqrt{1-h^{2}}\right) \sqrt{1-h^{2}}} \pi \\
& \quad \times\left[2 B_{03}-2 D_{30}+\left(B_{03}+B_{21}-D_{12}-D_{30}\right) \sqrt{1-h^{2}}\right. \\
& \left.\quad+2 D_{30} h^{2}\right] .
\end{aligned}
$$

Thus, $d T_{10}(h) / d h=-\left(2 \pi \widetilde{F}(\delta) / \delta^{3}(1+\delta)^{2}\right) \sqrt{1-\delta^{2}}$, where $\delta=\sqrt{1-h^{2}}, \widetilde{F}(\delta)=D_{30} \delta^{4}+2 D_{30} \delta^{3}+\left(D_{12}-B_{21}\right) \delta^{2}-$ $2 B_{03} \delta-B_{03}$. If $D_{30}=0, \widetilde{F}(\delta)=\left(D_{12}-B_{21}\right) \delta^{2}-2 B_{03} \delta-B_{03}$, we can control the coefficients $D_{12}-B_{21},-2 B_{03},-B_{03}$, which gives two zeros of $\widetilde{F}(\delta)$ in $(0,1)$. If $D_{30} \neq 0$, according to the expression of $\widetilde{F}(\delta)$, we suppose that $\delta_{1}, \delta_{2}, \delta_{3}, \delta_{4}$ are four zeros of $\widetilde{F}(\delta)$. From the relationship of root and coefficients, we have $\delta_{1}+\delta_{2}+\delta_{3}+\delta_{4}=-2$; thus not all of the zeros are in $(0,1)$. Then from [18], and it is impossible for $\widetilde{F}(\delta)$ to have 3 zeros in $(0,1)$. Thus, the first part of conclusion (i) is proved.

Next, we give an example to show that the zeros in $(0,1)$ can be achievable. We can choose $D_{30}=1300, D_{12}-B_{21}=$ $-2553, B_{03}=116$, then, $\widetilde{F}(\delta)=1300 \delta^{4}+2600 \delta^{3}-2553 \delta^{2}-$ $232 \delta-116=(2 \delta-1)(5 \delta-2)\left(130 \delta^{2}+377 \delta+58\right)$, so, $\widetilde{F}(\delta)$ has two simple zeros $2 / 5$ and $1 / 2$ in $(0,1)$. Thus, $T(h, \varepsilon, \lambda)$ has exactly two critical periods in $(0,1)$. The proof of conclusion (i) is completed.

For the conclusion (ii), if $M_{0}(h) \equiv 0$, we get $D_{30}=B_{03}=$ $D_{12}-B_{21}=0$ or $S_{2}(\theta)=0$.

Hence, from (26), we have

$$
\begin{aligned}
T_{11}(h)= & -\int_{0}^{2 \pi} r_{01}(\theta, h) S_{1}(\theta) d \theta-\int_{0}^{2 \pi} r_{00}(\theta, h) \bar{S}_{1}(\theta) d \theta \\
& -\int_{0}^{2 \pi} r_{00}^{2}(\theta, h) \bar{S}_{2}(\theta) d \theta-\int_{0}^{2 \pi} r_{00}^{3}(\theta, h) \bar{S}_{3}(\theta) d \theta \\
= & \frac{1-\sqrt{1-h^{2}}}{\left(1+\sqrt{1-h^{2}}\right) \sqrt{1-h^{2}}} \pi \\
& \times\left[2 \bar{B}_{03}-2 \bar{D}_{30}+\left(\bar{B}_{03}+\bar{B}_{21}-\bar{D}_{12}-\bar{D}_{30}\right)\right. \\
& \left.\quad \times \sqrt{1-h^{2}}+2 \bar{D}_{30} h^{2}\right] .
\end{aligned}
$$

So, $d T_{11}(h) / d h=-\left(2 \pi \tilde{\widetilde{F}}(\delta) / \delta^{3}(1+\delta)^{2}\right) \sqrt{1-\delta^{2}}$, where $\delta=$ $\sqrt{1-h^{2}}, \widetilde{F}(\delta)=\bar{D}_{30} \delta^{4}+2 \bar{D}_{30} \delta^{3}+\left(\bar{D}_{12}-\bar{B}_{21}\right) \delta^{2}-2 \bar{B}_{03} \delta-\bar{B}_{03}$. From the proof above, we can get result (ii). This ends the proof of Theorem 7.

Corollary 8. For system (58) with $a_{21}>0$, one obtains

$$
\begin{aligned}
T_{10}(h)= & \frac{1-\sqrt{1-a_{21} h^{2}}}{\left(1+\sqrt{1-a_{21} h^{2}}\right) \sqrt{1-a_{21} h^{2}}} \pi \\
& \times\left(2 b_{03}-2 c_{30}+\left(b_{03}+b_{21}-c_{12}-c_{30}\right)\right. \\
& \left.\quad \times \sqrt{1-a_{21} h^{2}}+2 c_{30} a_{21} h^{2}\right)\left(a_{21}\right)^{-1},
\end{aligned}
$$

where $h \in\left(0,1 / \sqrt{a_{21}}\right)$. For sufficiently small $\varepsilon$ and $\lambda$, there are at most two critical periods for $T(h, \varepsilon, \lambda)$ in $h \in(0,1)$ and the maximum number can be achievable.

Proof. Let $T(h, \varepsilon, \lambda)$ denote the period function of $(60)$ and $\bar{T}(\bar{h}, \varepsilon, \lambda)$ denote the period function of (58). Then we have $h=\sqrt{a_{21}} \bar{h}$, and $\left.T(h, \varepsilon, \lambda)\right|_{\varepsilon=\lambda=0}=\left.\bar{T}(\bar{h}, \varepsilon, \lambda)\right|_{\varepsilon=\lambda=0}$. Hence by Theorem 7 and (61), we have for system (58),

$$
\begin{aligned}
T_{10}(h)= & \frac{1-\sqrt{1-a_{21} h^{2}}}{\left(1+\sqrt{1-a_{21} h^{2}}\right) \sqrt{1-a_{21} h^{2}}} \pi \\
& \times\left(2 b_{03}-2 c_{30}+\left(b_{03}+b_{21}-c_{12}-c_{30}\right)\right. \\
& \left.\quad \times \sqrt{1-a_{21} h^{2}}+2 c_{30} a_{21} h^{2}\right)\left(a_{21}\right)^{-1},
\end{aligned}
$$

which gives the formula of $T_{10}(h)$. Other conclusions in the corollary can also be gotten from results in Theorem 7 similarly. This ends the proof of Corollary 8. 
Case $3(n=l=2, m=k=3)$. In this case, system (5) becomes

$$
\begin{gathered}
\dot{x}=-y+a_{11} x y+\lambda \bar{a}_{11} x y \\
+\varepsilon\left[b_{11} x y+b_{21} x^{2} y+b_{03} y^{3}\right. \\
\left.+\lambda\left(\bar{b}_{11} x y+\bar{b}_{21} x^{2} y+\bar{b}_{03} y^{3}\right)\right], \\
\dot{y}=x+a_{11} y^{2}+\lambda \bar{a}_{11} y^{2} \\
+\varepsilon\left[c_{20} x^{2}+c_{02} y^{2}+c_{30} x^{3}+c_{12} x y^{2}\right. \\
\left.\quad+\lambda\left(\bar{c}_{20} x^{2}+\bar{c}_{02} y^{2}+\bar{c}_{30} x^{3}+\bar{c}_{12} x y^{2}\right)\right] .
\end{gathered}
$$

Without loss of generality, we suppose $a_{11}=1$, otherwise, system (70) can be simplified as the form of system (71) by the transformation $u=a_{11} x, v=a_{11} y$. In this case, system (70) becomes

$$
\begin{gathered}
\dot{x}=-y+x y+\lambda \bar{a}_{11} x y \\
+\varepsilon\left[b_{11} x y+b_{21} x^{2} y+b_{03} y^{3}\right. \\
\left.+\lambda\left(\bar{b}_{11} x y+\bar{b}_{21} x^{2} y+\bar{b}_{03} y^{3}\right)\right], \\
\dot{y}=x+y^{2}+\lambda \bar{a}_{11} y^{2} \\
+\varepsilon\left[c_{20} x^{2}+c_{02} y^{2}+c_{30} x^{3}+c_{12} x y^{2}\right. \\
\left.+\lambda\left(\bar{c}_{20} x^{2}+\bar{c}_{02} y^{2}+\bar{c}_{30} x^{3}+\bar{c}_{12} x y^{2}\right)\right] .
\end{gathered}
$$

Theorem 9. For system (71) and sufficiently small $\varepsilon$ and $\lambda$, one has

(i)

$$
T_{10}(h)=-\pi\left(I_{1}+I_{2}\right)
$$

where

$$
\begin{gathered}
I_{1}=\left(c_{02}+b_{11}-3 c_{20}\right)+\frac{2\left(c_{02}+b_{11}-c_{20}\right)}{h^{2}}+\frac{4\left(c_{20}-c_{02}+b_{11}\right)}{h} \\
+\frac{2(1-h)\left[c_{20} h^{2}+2\left(c_{02}+b_{11}-c_{20}\right) h+c_{20}-c_{02}+b_{11}\right]}{h^{2} \sqrt{1-2 h}}, \\
I_{2}=\left(c_{30}-b_{03}-c_{12}+b_{21}\right) I_{21} \\
+\left(c_{12}-b_{21}+2 b_{03}\right) I_{22}-b_{03} I_{23}, \\
I_{21}=\left(-4 h^{6}-10 h^{5}+78 h^{4}-140 h^{3}-112 h^{2}\right. \\
-7(1-2 h)^{5 / 2} h^{2}+12(1-2 h)^{5 / 2} h \\
\left.-6(1-2 h)^{5 / 2}+6-42 h\right)\left((1-2 h)^{5 / 2} h^{2}\right)^{-1} \\
I_{22}=-2 \frac{2 h^{4}+h^{3}-7 h^{2}+5 h-1-(1-2 h)^{5 / 2}}{(1-2 h)^{5 / 2}} \\
I_{23}=\frac{2 h^{2}\left(4 h^{3}-8 h^{2}+5 h-1\right)}{(1-2 h)^{7 / 2}} .
\end{gathered}
$$

There can exist three critical periods for system (71) if $T_{10}(h)$ is not identically zero;

(ii) if $M_{10}(h)=T_{10}^{\prime}(h) \equiv 0$, one has

$$
T_{11}(h)=-\pi\left(I_{3}+I_{4}\right)
$$

where

$$
\begin{aligned}
I_{3}= & \left(\bar{c}_{02}-\bar{b}_{11}+3 \bar{c}_{20}\right)+\frac{2\left(\bar{c}_{02}-\bar{b}_{11}-\bar{c}_{20}\right)}{h^{2}} \\
& +\frac{4\left(\bar{c}_{20}-\bar{c}_{02}+\bar{b}_{11}\right)}{h} \\
& +2(1-h)\left[\bar{c}_{20} h^{2}+2\left(\bar{c}_{02}-\bar{b}_{11}-\bar{c}_{20}\right) h\right. \\
I_{4}= & \left.\left(\bar{c}_{30}-\bar{b}_{03}-\bar{c}_{12}+\bar{b}_{21}\right) I_{21}-\bar{c}_{02}+\bar{b}_{11}\right]\left(h^{2} \sqrt{1-2 h}\right)^{-1}, \\
& +\left(\bar{c}_{12}-\bar{b}_{21}+2 \bar{b}_{03}\right) I_{22}-\bar{b}_{03} I_{23} .
\end{aligned}
$$

There can appear three critical periods for system (71) if $T_{11}(h)$ is not identically zero.

Proof. From (13), (14), (22)-(25), we have

$$
\begin{gathered}
G_{0}(\theta)=\sin (\theta), \quad \bar{G}_{0}(\theta)=\bar{a}_{11} \sin (\theta), \\
G_{1}(\theta)=\left(c_{20}+b_{11}\right) \cos ^{2}(\theta) \sin (\theta)+c_{02} \sin ^{3}(\theta),
\end{gathered}
$$

$$
\begin{aligned}
G_{2}(\theta)= & \left(b_{21}+c_{30}\right) \cos ^{3}(\theta) \sin (\theta) \\
& +\left(b_{03}+c_{12}\right) \cos (\theta) \sin ^{3}(\theta),
\end{aligned}
$$

$S_{1}(\theta)=c_{20} \cos ^{3}(\theta)+\left(c_{02}-b_{11}\right) \cos (\theta) \sin ^{2}(\theta)$,

$$
\begin{aligned}
S_{2}(\theta)= & c_{30} \cos ^{4}(\theta)+\left(c_{12}-b_{21}\right) \cos ^{2}(\theta) \sin ^{2}(\theta) \\
& -b_{03} \sin ^{4}(\theta)
\end{aligned}
$$

$$
\begin{gathered}
\bar{G}_{1}(\theta)=\left(\bar{b}_{11}+\bar{c}_{20}\right) \cos ^{2}(\theta) \sin (\theta)+\bar{c}_{02} \sin ^{3}(\theta), \\
\bar{G}_{2}(\theta)=\left(\bar{b}_{21}+\bar{c}_{30}\right) \cos ^{3}(\theta) \sin (\theta) \\
+\left(\bar{b}_{03}+\bar{c}_{12}\right) \cos (\theta) \sin ^{3}(\theta), \\
\bar{S}_{1}(\theta)=\bar{c}_{20} \cos ^{3}(\theta)+\left(\bar{c}_{02}-\bar{b}_{11}\right) \cos (\theta) \sin ^{2}(\theta), \\
\bar{S}_{2}(\theta)=\bar{c}_{30} \cos ^{4}(\theta)+\left(\bar{c}_{12}-\bar{b}_{21}\right) \cos ^{2}(\theta) \sin ^{2}(\theta) \\
-\bar{b}_{03} \sin ^{4}(\theta) .
\end{gathered}
$$


From (10), we can get

$$
\begin{aligned}
& T_{10}(h)=-\int_{0}^{2 \pi} \frac{h S_{1}(\theta)}{1-h+h \cos (\theta)} d \theta \\
&-\int_{0}^{2 \pi} \frac{h^{2} S_{2}(\theta)}{(1-h+h \cos (\theta))^{2}} d \theta \\
&=-\int_{0}^{2 \pi}\left(\left(h \left(c_{20} \cos ^{3}(\theta)+\left(c_{02}-b_{11}\right)\right.\right.\right. \\
&\left.\times\left(1-h+h \cos (\theta) \sin ^{2}(\theta)\right)\right) \\
&-\int_{0}^{2 \pi}\left(\left(h ^ { 2 } \left[c_{30} \cos ^{4}(\theta)+\left(c_{12}-b_{21}\right) d \theta\right.\right.\right. \\
&\left.\left.\times \cos ^{2}(\theta) \sin ^{2}(\theta)-b_{03} \sin ^{4}(\theta)\right]\right) \\
&\left.\times\left(1-h+h \cos ^{-1}(\theta)\right)^{-2}\right) d \theta \\
&=-\pi\left(I_{1}+I_{2}\right),
\end{aligned}
$$

where

$$
\begin{aligned}
& I_{1}=\left(c_{02}-b_{11}-3 c_{20}\right)+\frac{2\left(c_{02}-b_{11}-c_{20}\right)}{h^{2}} \\
& +\frac{4\left(c_{20}-c_{02}+b_{11}\right)}{h} \\
& +2(1-h)\left[c_{20} h^{2}+2\left(c_{02}-b_{11}-c_{20}\right) h\right. \\
& \left.+c_{20}-c_{02}+b_{11}\right]\left(h^{2} \sqrt{1-2 h}\right)^{-1}, \\
& I_{2}=\left(c_{30}-b_{03}-c_{12}+b_{21}\right) I_{21} \\
& +\left(c_{12}-b_{21}+2 b_{03}\right) I_{22}-b_{03} I_{23}, \\
& I_{21}=\left(-4 h^{6}-10 h^{5}+78 h^{4}-140 h^{3}-112 h^{2}\right. \\
& -7(1-2 h)^{5 / 2} h^{2}+12(1-2 h)^{5 / 2} h \\
& \left.-6(1-2 h)^{5 / 2}+6-42 h\right) \\
& \times\left((1-2 h)^{5 / 2} h^{2}\right)^{-1} \\
& I_{22}=-2 \frac{2 h^{4}+h^{3}-7 h^{2}+5 h-1-(1-2 h)^{5 / 2}}{(1-2 h)^{5 / 2}} \text {, } \\
& I_{23}=\frac{2 h^{2}\left(4 h^{3}-8 h^{2}+5 h-1\right)}{(1-2 h)^{7 / 2}} \text {. }
\end{aligned}
$$

By the same method as the proof of Theorem 6, we have conclusion (i).
For conclusion (ii), if $M_{10}(h)=T_{10}^{\prime}(h) \equiv 0$, giving $S_{1}(\theta)=$ $S_{2}(\theta)=0$, thus from $(26)$,

$$
\begin{aligned}
& T_{11}(h)=-\int_{0}^{2 \pi} r_{00}(\theta, h) \bar{S}_{1}(\theta) d \theta \\
&-\int_{0}^{2 \pi} r_{00}^{2}(\theta, h) \bar{S}_{2}(\theta) d \theta \\
&=-\int_{0}^{2 \pi}\left(\left(h \left[\bar{c}_{20} \cos ^{3}(\theta)\right.\right.\right. \\
&\left.\left.+\left(\bar{c}_{02}-\bar{b}_{11}\right) \cos (\theta) \sin ^{2}(\theta)\right]\right) \\
&\left.\times(1-h+h \cos (\theta))^{-1}\right) d \theta \\
&-\int_{0}^{2 \pi}\left(\left(h ^ { 2 } \left[\bar{c}_{30} \cos ^{4}(\theta)+\left(\bar{c}_{12}-\bar{b}_{21}\right)\right.\right.\right. \\
&\left.\left.\times \cos ^{2}(\theta) \sin ^{2}(\theta)-\bar{b}_{03} \sin ^{4}(\theta)\right]\right) \\
&\left.\quad \times(1-h+h \cos (\theta))^{-2}\right) d \theta \\
&=-\pi\left(I_{3}+I_{4}\right),
\end{aligned}
$$

where

$$
\begin{aligned}
I_{3}= & \left(\bar{c}_{02}-\bar{b}_{11}+3 \bar{c}_{20}\right)+\frac{2\left(\bar{c}_{02}-\bar{b}_{11}-\bar{c}_{20}\right)}{h^{2}} \\
& +\frac{4\left(\bar{c}_{20}-\bar{c}_{02}+\bar{b}_{11}\right)}{h} \\
& +2(1-h)\left[\bar{c}_{20} h^{2}+2\left(\bar{c}_{02}-\bar{b}_{11}-\bar{c}_{20}\right) h\right. \\
I_{4}= & \left(\bar{c}_{30}-\bar{b}_{03}-\bar{c}_{12}+\bar{b}_{21}\right) I_{21} \\
& +\left(\bar{c}_{12}-\bar{b}_{21}+2 \bar{b}_{03}\right) I_{22}-\bar{b}_{03} I_{23} .
\end{aligned}
$$

We can get conclusion (ii) with the same method as the proof of Theorem 6 . This ends the proof of Theorem 9.

\section{Acknowledgment}

The project was supported by National Natural Science Foundation of China (11271261).

\section{References}

[1] L. P. Bonorino, E. H. M. Brietzke, J. P. Lukaszczyk, and C. A. Taschetto, "Properties of the period function for some Hamiltonian systems and homogeneous solutions of a semilinear elliptic equation," Journal of Differential Equations, vol. 214, no. 1, pp. 156-175, 2005.

[2] C. Chicone, "The monotonicity of the period function for planar Hamiltonian vector fields," Journal of Differential Equations, vol. 69 , no. 3, pp. 310-321, 1987. 
[3] S.-N. Chow and J. A. Sanders, "On the number of critical points of the period," Journal of Differential Equations, vol. 64, no. 1, pp. 51-66, 1986.

[4] S.-N. Chow and D. Wang, "On the monotonicity of the period function of some second order equations," Ćasopis Pro Pěstování Matematiky, vol. 111, no. 1, pp. 14-25, 1986.

[5] A. Cima, A. Gasull, and F. Mañosas, "Period function for a class of Hamiltonian systems," Journal of Differential Equations, vol. 168, no. 1, pp. 180-199, 2000.

[6] E. Freire, A. Gasull, and A. Guillamon, "First derivative of the period function with applications," Journal of Differential Equations, vol. 204, no. 1, pp. 139-162, 2004.

[7] J. Smoller and A. Wasserman, "Global bifurcation of steadystate solutions," Journal of Differential Equations, vol. 39, no. 2, pp. 269-290, 1981.

[8] J. Waldvogel, "The period in the Lotka-Volterra system is monotonic," Journal of Mathematical Analysis and Applications, vol. 114, no. 1, pp. 178-184, 1986.

[9] C. Chicone and F. Dumortier, "Finiteness for critical periods of planar analytic vector fields," Nonlinear Analysis: Theory, Methods \& Applications, vol. 20, no. 4, pp. 315-335, 1993.

[10] P. de Maesschalck and F. Dumortier, "The period function of classical Liénard equations," Journal of Differential Equations, vol. 233, no. 2, pp. 380-403, 2007.

[11] J. Chavarriga and M. Sabatini, "A survey of isochronous centers," Qualitative Theory of Dynamical Systems, vol. 1, no. 1, pp. 1-70, 1999.

[12] W. S. Loud, "Behavior of the period of solutions of certain plane autonomous systems near centers," Contributions to Differential Equations, vol. 3, pp. 21-36, 1964.

[13] I. I. Pleshkan, "On isochronicity conditions of the system of two differential equations," Journal of Differential Equations, vol. 4, pp. 1991-1993, 1968.

[14] C. Chicone and M. Jacobs, "Bifurcation of critical periods for plane vector fields," Transactions of the American Mathematical Society, vol. 312, no. 2, pp. 433-486, 1989.

[15] A. Cima, A. Gasull, and P. R. da Silva, "On the number of critical periods for planar polynomial systems," Nonlinear Analysis: Theory, Methods \& Applications, vol. 69, no. 7, pp. 1889-1903, 2008.

[16] A. Gasull and J. Yu, "On the critical periods of perturbed isochronous centers," Journal of Differential Equations, vol. 244, no. 3, pp. 696-715, 2008.

[17] A. Gasull and Y. Zhao, "Bifurcation of critical periods from the rigid quadratic isochronous vector field," Bulletin des Sciences Mathématiques, vol. 132, no. 4, pp. 292-312, 2008.

[18] X. Chen, V. G. Romanovski, and W. Zhang, "Critical periods of perturbations of reversible rigidly isochronous centers," Journal of Differential Equations, vol. 251, no. 6, pp. 1505-1525, 2011.

[19] J.-P. Françoise, "The successive derivatives of the period function of a plane vector field," Journal of Differential Equations, vol. 146, no. 2, pp. 320-335, 1998.

[20] E. Freire, A. Gasull, and A. Guillamon, "Period function for perturbed isochronous centres," Qualitative Theory of Dynamical Systems, vol. 3, no. 1, pp. 275-284, 2002. 


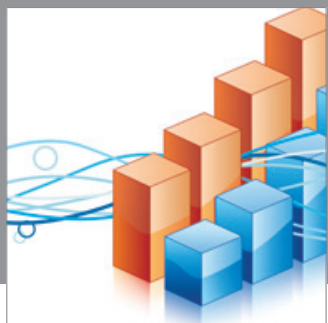

Advances in

Operations Research

mansans

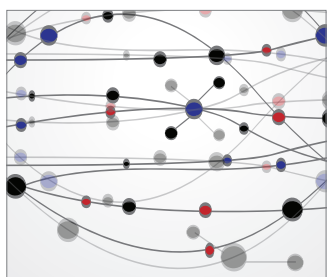

The Scientific World Journal
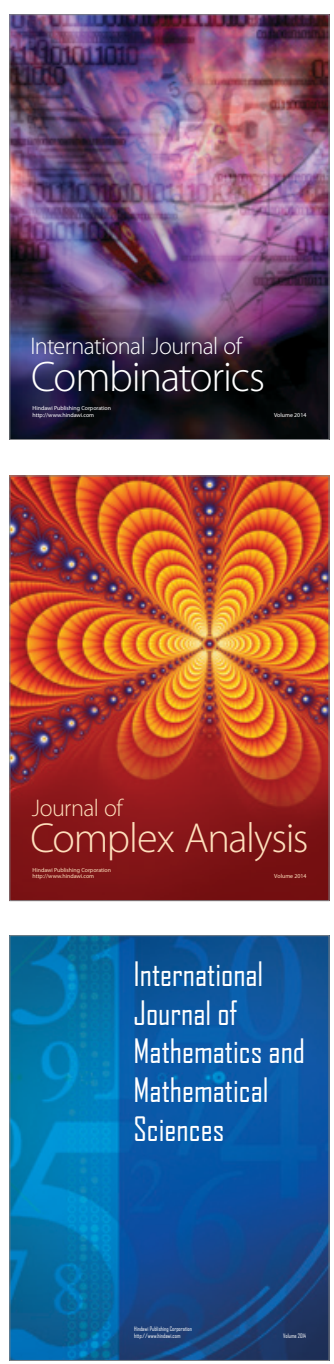
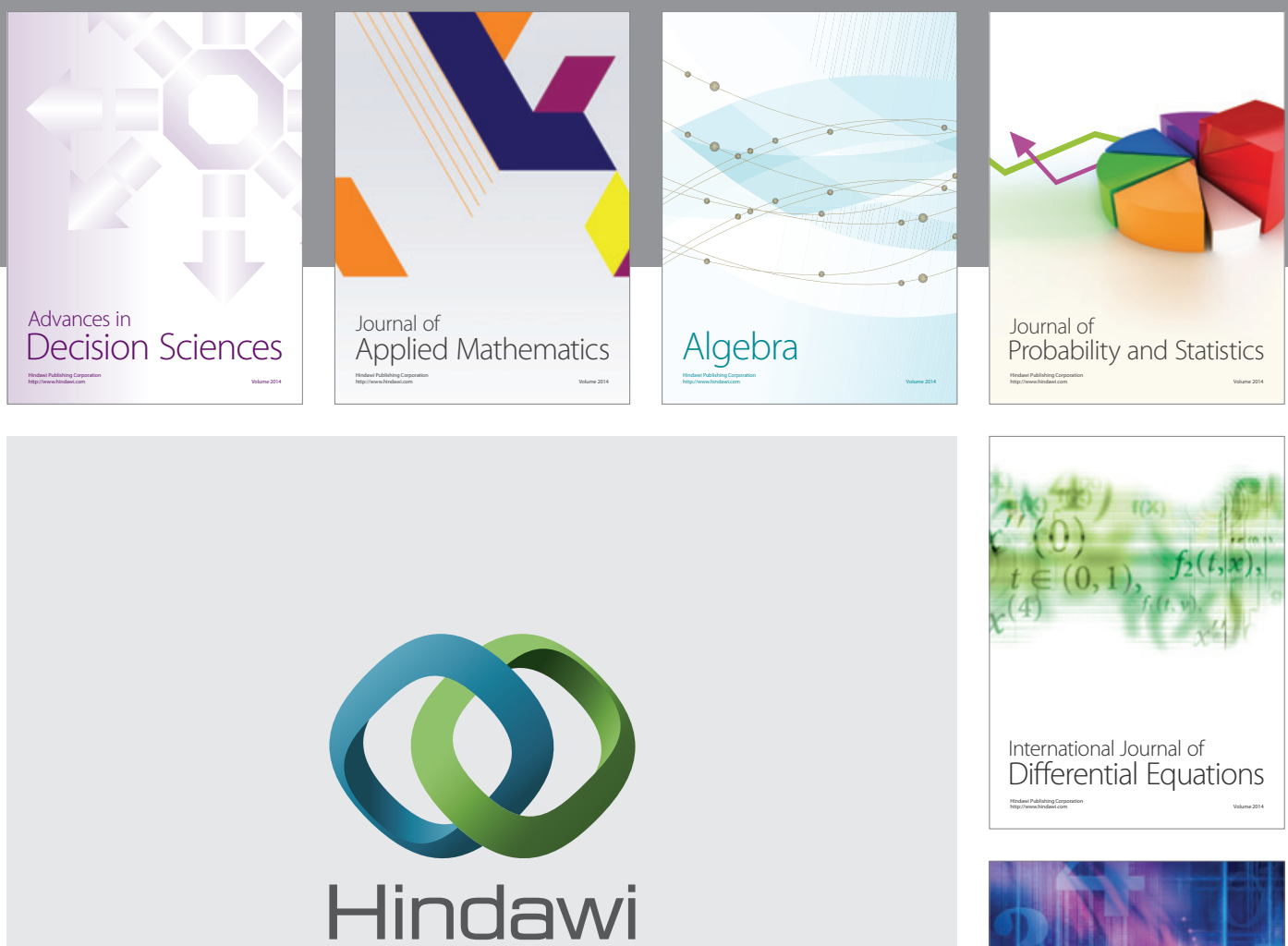

Submit your manuscripts at http://www.hindawi.com
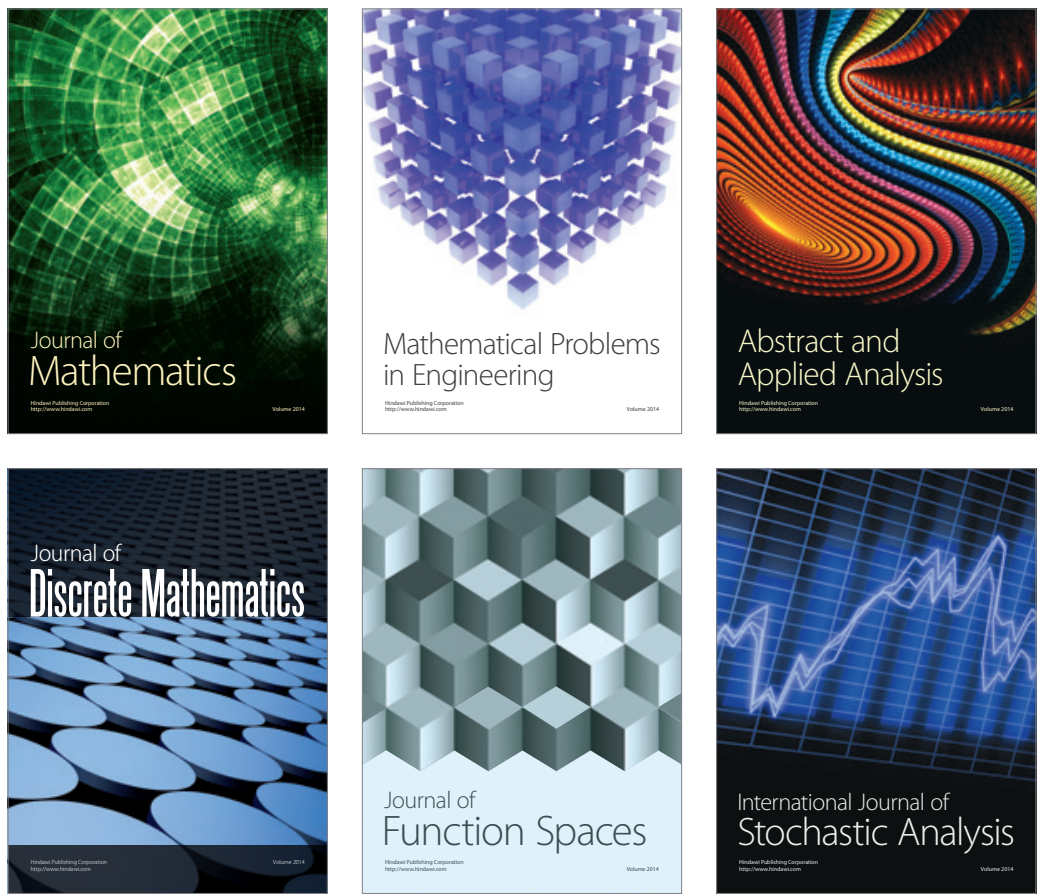

Journal of

Function Spaces

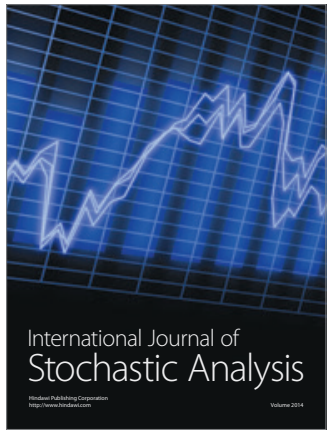

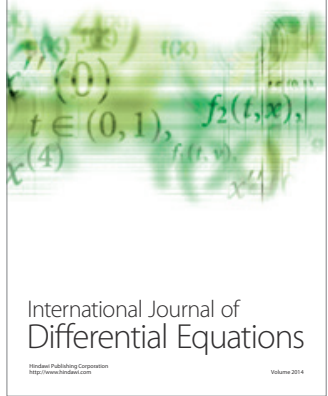
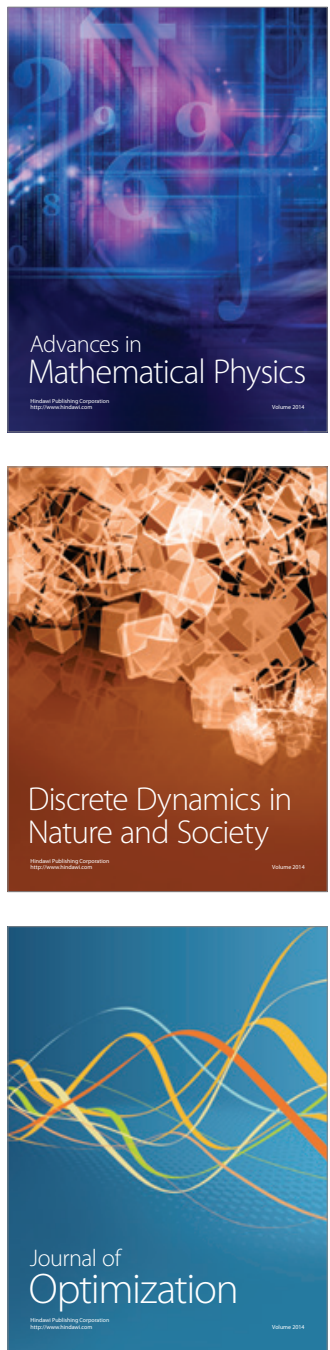